Годишњак Филозофског факултета у Новом Саду, Кюига ХХХVII (2012)

Annual Review of the Faculty of Philosophy, Novi Sad, Volume XXXVII (2012)

Gordana Ristić

UDC 811.112.2’373.7:811.163.41’373.7

Filozofski fakultet Univerziteta u Novom Sadu

Originalan naučni rad

goca33@yahoo.de

\title{
LIEBE IN DEUTSCHEN UND SERBISCHEN SOMATISCHEN PHRASEOLOGISMEN
}

Das Ziel dieses Beitrags ist es, auf der Grundlage der kognitiven linguistischen Theorie eine vergleichende Untersuchung von somatischen Phraseologismen im Deutschen und Serbischen, die im semantischen Feld LIEBE vorkommen, durchzuführen. Somatismen werden aus interlingualer deutsch-serbischer Perspektive untersucht, wobei das Deutsche als Ausgangsgsprache und das Serbische als Zielsprache betrachtet werden. Als Grundlage für das Exzerpieren des untersuchten Materials wurden entsprechende phraseologische Wörterbücher beider Sprachen benutzt.

Schlüsselwörter: Phraseologismen, Emotion, Liebe, kontrastive Analyse, konzeptuelle Metapher.

\section{EINFÜHRUNG}

In diesem Beitrag wird ein Teilbereich des phraseologiereichen semantischen Feldes der Emotionen dargestellt. Den Untersuchungsgegenstand bilden deutsche und serbische somatische Phraseologismen des semantischen Feldes LIEBE mit bildlich-metaphorischer und symbolischer Motiviertheit. Somatismen werden aus interlingualer deutsch-serbischer Perspektive untersucht, wobei das Deutsche als Ausgangsgsprache und das Serbische als Zielsprache betrachtet werden. Das Ziel des Beitrags ist es, auf der Grundlage der kognitiven linguistischen Theorie eine kontrastive Analyse von somatischen Phraseologismen im Deutschen und Serbischen, die im semantischen Feld LIEBE vorkommen, durchzuführen. Die metaphorischen Konzepte der Liebe werden auch auf kontrastiver Ebene untersucht. Bei der Zuordnung der Somatismen zu den konzeptuellen Metaphern wird Theorie der konzeptuellen Metaphern von Lakoff und Johnson als Grundlage genommen.

Das untersuchte Material wurde aus entsprechenden phraseologischen Wörterbüchern beider Sprachen exzerpiert. 
Viele metaphorische Phraseologismen basieren auf metaphorischen Konzepten, denen als Herkunftsbereich unser Körper dient. Somatismen sind feste polylexikalische sprachliche Einheiten, die eine Körperteilbezeichnung als Konstituente haben. Sie beruhen auf der traditionellen Symbolik der Körperteile, auf Gesten und Mimik des Menschen, auf allgemein verbreiteten Volkstraditionen und Aberglauben. Als universelles Phänomen sind sie in allen Sprachen zu finden und gehören zum Grundwortschatz. Sie stellen auch einen essentiellen Bestandteil der Emotions-Phraseologismen dar, weil sie zur Schilderung der Emotionen geeignet sind. Viele Emotionen werden durch Phraseologismen konzeptualisiert, so dass der Metapher eine wichtige Rolle bei der Konzeptbildung zukommt. Nach Földes ,dienen Somatismen gewöhnlich als Ausdruck von emotionalen, mentalen Eigenschaften und verschiedenen Handlungen des Menschen, widerspiegeln sein Verhältnis zur Umwelt und drücken die traditionelle Symbolik der Körpersprache, so wie lokale oder allgemein verbreitete Traditionen und Aberglauben aus“"(Földes 1985:21).

Für das menschliche Leben sind Emotionen konstitutive Phänomene. Sie ,steuern maßgeblich unsere Denk- und Handlungsprozesse, determinieren die Interpretation und Evaluation von Mitmenschen und Situationen, erleichtern, erschweren oder hemmen unsere Lernprozesse und haben auf unsere Erinnerungsprozesse einen erheblichen Einfluss“" (Schwarz-Friesel 2007:4).

Die Liebe bezeichnet die intensivste Empfindung und lässt sich als eine innere Einstellung positiver, tiefer Verbundenheit zu einer Person beschreiben. Sie stellt die stärkste Form der Hinwendung zu einem anderen Lebewesen dar. Die Liebe wird entweder als universale Emotion oder als Kulturmuster, als symbolischer Kommunikationscode beschrieben. Unsere Vorstellung von romantischer Liebe wird von bestimmten, kulturell und literaturhistorisch geprägten Konzeptualisierungsund Kommunikationsmustern bestimmt. Davon zu unterscheiden sind jeweils kulturspezifische, gesellschaftliche Konzeptualisierungen von „Liebe“ als Gefühl, die eine kognitive Beurteilung und Klassifikation beinhaltet (Schwarz-Friesel 2007: 288-310).

Eine große Zahl von Phraseologismen und Metaphern weisen darauf hin, wie eng emotionale Zustände mit körperlichen Zuständen verbunden sind. Emotionen sind abstrakt, sie müssen also aus der unsichtbaren, unfassbaren Welt des Geistes in die sichtbare, objektivierbare Welt herüberholt werden (Lakoff/Johnson 2007: 73; 
Dobrovolskij 1997: 172).

Bei der Versprachlichung der Emotionen spielen Phraseologismen und Metaphern eine besondere Rolle, da die emotionale Sphäre nicht direkt zugänglich ist. Unser metaphorisch strukturiertes Konzeptsystem basiert auf unserer Erfahrung (Lakoff/Johnson 2007: 71-74).

Lakoff und Johnson, Vertreter der kognitiven Metapherntheorie, sind der Meinung, dass abstraktere Begriffe mittels metaphorischer Projektion durch weniger abstrakte Begriffe verstanden werden. Metaphern gelten als sprachliche Mittel der Konzeptualisierung. Nach Palm (Palm 1997:1) werden mentale Größen wie Emotionen durch Phraseologismen konzeptualisiert. Phraseologismen entstehen aus einem Konzept, aus einer gedanklichen Repräsentation einer Kategorie. Nach Lakoff und Johnson (2007:11) ist unser alltägliches Konzeptsystem im Kern und grundsätzlich metaphorisch.

Das Wesen der Metapher besteht darin „dass wir durch sie eine Sache oder einen Vorgang in Begriffen einer anderen Sache, bzw. eines anderen Vorgangs verstehen und erfahren können.“ Jede Metapher hat einen Quellenbereich (source), einen Zielbereich (target) und einen Übertragungsvorgang (to mapping) (Lakoff 1987:276).

Die körperlichen Erfahrungen sind als Herkunftsbereich zur metaphorischen Struktuierung eines abstrakten Zielbereichs stets vorhanden.

\section{KONZEPTUELLE METAPHERN IM SEMANTISCHEN FELD LIEBE}

In der kognitiven Linguistik wird das Konzept der LIEBE als zentraler Punkt in einem Netzwerk verschiedener Kategorien von Konzepten wie FREUDE, GLÜCK, SEXUELLE BEGIERDE etc. gesehen werden (s.z.B.Kövecses 1999:44f.). Es besteht eine Kausalbeziehung zwischen GLÜCK und LIEBE (Kövecses 1999:141). ${ }^{1}$ Die Emotion Liebe wird in diesem Beitrag als ein semantisches Feld behandelt.

Phraseologismen des gleichen semantischen Feldes können aufgrund

1 Das Konzept der Liebe ist mit dem Konzept MÖGEN, SCHÖNHEIT /ATTRAKTIVITÄT unmittelbar verbunden. Kövecses Untersuchungen zur Metaphorisierung des Konzeptes LIEBE fokussieren besonders die Konzeptualisierung EMOTION IST PHYSIOLOGISCHE REAKTION (z.B. Veränderung des Herzschlags, des Atmens und der Gesichtfarbe; Kövecses 1999: 172f). 
einer beschränkten Anzahl konzeptueller Metaphern beschrieben werden. Im mentalen Lexikon sind sie als struktuierte Cluster gespeichert und nach der Logik der konzeptuellen Metapher miteinander vernetzt. Semantisch verwandte Phraseologismen, die verschiedenen Herkunftsbereichen angehören, sind in einem Feld zu finden (Dobrovolskij 1997: 30). Die Liebe wird in dieser Arbeit als Hyperonym für verschiedene Ausprägungen dieser Emotion gebraucht, die von Zuneigung, Sympathie, bis hin zur Liebe ${ }^{2}$ reicht. Es wurde schon betont, dass viele Somatismen Emotionen ausdrücken. Das Konzept LIEBE, das in deutschen und serbischen Phraseologismen realisiert wird, basiert auf konzeptuellen Metaphern mit der Konstituente $\mathrm{Herz}^{3}$. Viele metaphorische Konzepte, die den Herz-Somatismen als Grundlage dienen, haben als Zielbereich dieEmotion Liebe. Dieser Somatismus vertritt den Sitz der Emotionen und ist stärker als andere Organe mit Emotionen verbunden. Fast in allen Sprachen wird das Herz (vgl. Sadikaj 2010) als Entstehungsplatz und Behälter der Emotionen betrachtet. Phraseologismen des semantischen Feldes LIEBE lassen sich aufgrund folgenden konzeptueller Metaphern beschreiben:

\subsection{LIEBE IST EIN OBJEKT}

Emotionen werden auch als Objekte konzeptualisiert und zwar durch ontologische Metaphern. Durch diese Metaphern ist uns unsere Erfahrung erst erfassbar, sie ermöglichen die Konkretisierung der mehr oder weniger abstrakten Vorstellungen. Unser eigener Körper ist die wichtigste Quelle der ontologischen Metaphern, so dass viele Ereignisse, Handlungen, Emotionen usw. oft als ein Teil unseres eigenen Körpers verstanden werden und als Objekte konzeptualisiert werden. Die bekannteste Metapher dieser Art ist die Gefäß/Container-Metapher. Abstrakte Konzepte können also als Substanz wahrgenommen werden (z.B. ÄRGER IST HEISSE SUBSTANZ). Den offensichtlichsten ontologischen Charakter unter diesen Metaphern hat die Personifikation, bei der physische Objekte in Gestalt einer Person näher spezifiziert werden (vgl. Lakoff/Johnson 2007: 35-44; Dobrovolskij 1997: 27 32).

2 Liebe wird oft als, WÄRME/FEUER, LICHT / HELLIGKEIT, REISE, KRANKHEIT /WAHNSINN; PHYSIOLOGISCHE REAKTION, KRAFT/NATURKRAFT/MACHT, ORGANISMUS etc. konzeptualisiert. 


\subsection{LIEBE IST EIN WERTVOLLES OBJEKT IN EINEM GEFÄSS}

Die Liebe wird als etwas Wertvolles in einem Gefäß konzeptualisiert. Der Somatismus Herz, der im Herkunftsbereich ist, wird hier als Gefäß betrachtet. Die Gefäß/Container-Metapher ist eine der grundlegenden Metaphern bei der menschlichen Wahrnehmung der Welt (Lakoff/Johnson 2003: 29). Im Schrein wurden sehr oft wertvolle Dinge verschlossen. Der Phraseologismus geht vom Bild des Herzens als Schrein aus, in dem man jemanden einschließt und ganz mit seiner Liebe und Zuneigung umgibt. Der Vergleich des Herzens mit einem Schrein und das dazugehörige Bild vom Herzensschlüssel spielt in Volkslied, Märchen usw. eine große Rolle (Röhrich, L. 1991-1992: 706). Die Metaforik zu Gefäß oder Schrein ist besonders im Bereich der Lyrik anzutreffen ${ }^{4}$ (Müller 2005: 252).

\begin{tabular}{|l|l|l|}
\hline Deutsch & Serbisch & Äquivalenz \\
\hline jmdn in sein Herz schließen & naći nekome mesta u srcu & Teiläquivalenz \\
\hline jmdn im Herzen tragen & nositi/imati na/u srcu nekoga & Volläquivalenz \\
\hline$/$ & biti/ležati kome u srcu & Nulläquivalenz \\
\hline
\end{tabular}

Beim ersten Phraseologismus ist Teiläquivalenz festgestellt. Im Serbischen wurde der Phraseologismus mit dem Verb „schließen“ nicht bestätigt. Wenn man jemanden liebt, dann wird für ihn „ein Platz im Herzen gefunden“.

Ins Herz wird jemand, der für uns sehr wichtig ist, geschlossen und im Herzen getragen, so dass das Herz als Gefäß für ein wertvolles Objekt betrachtet wird. Dieses Objekt kann sowohl etwas Konkretes als auch etwas Abstraktes sein.

Im Serbischen sind noch zwei Phraseologismen bestätigt biti/ležati kome u $s r c u$, die keine äquivalenten Phraseologismen im Deutschen haben. Im Deutschen besteht Phraseologismus jmdm am Herzen liegen mit der Bedeutung jmds innerstes Anliegen sein (Duden 11 1998:330), etwas ist für jmdn sehr wichtig (Müller 2005: 254; Schemann 1991: 207).

$4 \quad$ Vgl. Mhd. Du bist mein, ich bin dein, dessen kannst du sicher sein, du bist eingeschlossen in meinem Herzen. 


\subsection{LIEBE IST ETWAS VERSTECKTES/EIN VERSTECKTES OBJEKT IN EINEM GEFÄSS}

Der Somatismus Herz, durch den die Liebe metaphorisiert wird, wird auch hier als Gefäß betrachtet. Die Gefäßmetapher ist stark vertreten in den EmotionsPhraseologismen.

In der Natur des Menschen liegt es, seine Emotionen zu verstecken, weil sie als etwas Wertvolles gelten. Sie werden nur von denjenigen, die uns nahe stehen, für die wir Zuneigung, Sympathie und Liebe empfinden, entdeckt werden. Sein Herz für jemanden entdecken, bedeutet, sich der Liebe, seiner Sympathie für jemanden oder seines Interesses bewusst werden.

\begin{tabular}{|l|l|l|}
\hline Deutsch & Serbisch & Äquivalenz \\
\hline $\begin{array}{l}\text { jmdm sein Herz öffnen /eröffnen / } \\
\text { aufschließen }\end{array}$ & $\begin{array}{l}\text { otvoriti/rastvoriti [svoje] srce } \\
\text { nekome }\end{array}$ & Volläquivalenz \\
\hline sein Herz für jmdn entdecken & $\begin{array}{l}\text { otvoriti/rastvoriti [svoje] srce } \\
\text { nekome }\end{array}$ & Teiläquivalenz \\
\hline
\end{tabular}

\subsection{LIEBE IST EIN GESCHENK}

Durch den Somatismus Herz als Geschenk wird die Liebe metaphorisiert. Unter dem Konzept Geschenk versteht man ein kostbares und wertvolles Objekt. Das Schenken des Herzens als Symbol der Liebe ist ziemlich alt. Jemandem das Herz schenken bedeutet ihm seine Zuneigung und Liebe geben.

\begin{tabular}{|l|l|l|}
\hline Deutsch & Serbisch & Äquivalenz \\
\hline jmdm sein Herz schenken & pokloniti [svoje] srce nekome & Volläquivalenz \\
\hline
\end{tabular}

Bei diesem Beispiel wird Volläquivalenz festgestellt, weil Phraseologismen bezüglich aller wesentlicher Äquivalenzparameter miteinander übereinstimmen.

\subsection{LIEBE IST DAS STEHLEN EINES KOSTBAREN OBJEKTS}

Das Konzept der Liebe wird durch den Somatismus Herz als Dieb metaphorisiert. Dieses metaphorische Konzept ist in vielen Sprachen zu finden. 
„Nach 2. Sam.12,5 sagen wir jemandem das Herz stehlen und nennen den, der es tut, einen Herzensdieb (Röhrich 1991-1992: 707).“ Wenn jemandem das Herz gestohlen wird, dann wird seine Liebe gewonnen.

\begin{tabular}{|l|l|l|}
\hline Deutsch & Serbisch & Äquivalenz \\
\hline jmdm sein Herz stehlen & *ukrasti srce nekome & Teiläquivalenz \\
\hline
\end{tabular}

In serbischen Wörterbüchern ist dieser Phraseologismus nicht belegt, aber in der Umgangssprache tritt er häufig vor.

\subsection{LIEBE IST EIN OBJEKT ZUM EROBERN}

Hier wird die Liebe als ein Objekt zum Erobern konzeptualisiert. Diese konzeptuelle Metapher ist anthropologisch begründet: sie beruht auf der Tatsache, dass Menschen immer neue Territorien erobern möchten. Jemandes Herz erobern bedeutet jemandes Sympathie schnell gewinnen. Die Konzeptualisierung manifestiert sich nur im Somatismus Herz in beiden Sprachen:

\begin{tabular}{|l|l|l|}
\hline Deutsch & Serbisch & Äquivalenz \\
\hline $\begin{array}{l}\text { jmds Herz/alle Herzen/die } \\
\text { Herzen im Sturm erobern }\end{array}$ & osvojiti nečije srce & Teiläquivalenz \\
\hline
\end{tabular}

Bei diesem Beispiel wird Teiläquivalenz festgestellt, weil im Serbischen das Lexem Sturm nicht vertreten ist, so dass der Phraseologismus im Deutschen auf der semantischen Ebene stärker ausgeprägt ist. Es muss auch betont werden, dass in diesem Fall zwei Metaphern vernetzt sind. Liebe kann hier auch als Krieg betrachtet werden- LIEBE IST KRIEG (vgl. Lakoff J. 2007: 63). ${ }^{5}$

\subsection{LIEBE IST DER FLUG EINES VOGELS}

Es ist nicht selten, dass unsere Emotionen, die wachsen, kommen, gehen, fliegen usw., als ein Lebewesen vorgestellt werden. Das Herz steht hier für Zuneigung 5 Die Liebe erscheint auch als Krieg, als Gegner, als eine Naturgewalt, physische Kraft, als Magie, auch als Wahnsinn. 
und Sympathie. In diesem Phraseologismus wird der Somatismus Herz aus dem Herkunftsbereich mit einem Vogel verglichen, so dass die Liebe als Vogel, aber auch als Flug metaphorisiert werden kann. Der Flug stellt hier die Liebe als positive Emotion dar.

\begin{tabular}{|l|l|l|}
\hline Deutsch & Serbisch & Äquivalenz \\
\hline jmdm fliegen alle/die Herzen zu & $/$ & Nulläquivalenz \\
\hline
\end{tabular}

Im Duden (Duden11 1998:326) ist die Semantik dieses Phraseologismus auf folgende Weise erklärt: ,jemand ist sehr beliebt, so dass er die Menschen für sich einnimmt.“ Nach Müller (Müller 2005:254) bedeutet dieser Phraseologismus, dass „das Gefühl der Liebe in vielen Wendungen mit dem verschenkten, verlorenen oder gebrochenen Herzen gleichgesetzt wird. In diesem Bild ist auch die Redensart von Herzen angesiedelt, das jemandem zufliegt, weil der Verliebte sein Herz verlor".

Das ist ein Beispiel für die Nulläquivalenz, weil im Serbischen kein entsprechender Phraseologismus belegt ist.

\subsection{LIEBE IST EINE PFLANZE/BLUME}

In folgenden Phraseologismen wird die Liebe durch semantische Transposition des Somatismus Herz als Pflanze konzeptualisiert:

\begin{tabular}{|l|l|l|}
\hline Deutsch & Serbisch & Äquivalenz \\
\hline $\begin{array}{l}\text { den Keim der Liebe in jmds } \\
\text { Herz senken }\end{array}$ & $\begin{array}{l}\text { zasejati/posaditi seme/klicu } \\
\text { ljubavi u nečije srce }\end{array}$ & Volläquivalenz \\
\hline $\begin{array}{l}\text { einem/jmdm ans Herz } \\
\text { gewachsen sein }\end{array}$ & $\begin{array}{l}\text { prirasti } \text { krcu } \text { (za srce) } \\
\text { nekome }\end{array}$ & Volläquivalenz \\
\hline$/$ & srce mi vene i kopni za nekim & Nulläquivalenz \\
\hline
\end{tabular}

Zwei Phraseologismen aus dieser Gruppe zeigen keine kontrastiven Unterschiede, aber im Serbischen haben wir auch ein Beispiel für Nulläquivalenz, wobei die Pflanze starke Sehnsucht und Liebe metaphorisiert. 
Das Spiel ist ein beliebter Ausgangsbereich bei der Konzeptualisierung des menschlichen Lebens überhaupt ${ }^{6}$, auch beim Konzept der LIEBE, weil die Sprecher sowohl das Leben als auch die Liebe als ein Glücksspiel auffassen. Die SpielMetapher ist auch als Strukturprinzip eines Zielbereichs der Liebe belegt.

\subsubsection{LIEBE IST VERLIEREN/GEWINNEN}

Bei diesem Glücksspiel kann man gewinnen oder verlieren, so dass die Liebe als Verlust der Kontrolle über eigene Emotionen, aber auch als Gewinn dargestellt wird.

\begin{tabular}{|l|l|l|}
\hline Deutsch & Serbisch & Äquivalenz \\
\hline sein Herz (an jmdn) verlieren & izgubiti glavu (zaljubiti se) & Teiläquivalenz \\
\hline jmds Herz gewinnen & zadobiti/osvojiti nečije srce & Volläquivalenz \\
\hline
\end{tabular}

Der serbische Phraseologismus zadobiti/osvojiti nečije srce wurde in entsprechenden Wörterbüchern nicht belegt, aber es muss betont werden, dass er ein Teil der Umgangssprache ist.

\subsection{LIEBE IST FEUER}

Emotionen und Gefühle werden oft als verschiedene Energiequellen konzeptualisiert. Was die Konzeptualisierung der Liebe als Feuer anbelangt, legt Kövesces dar, dass wir uns eine verliebte Person, bei der Verwendung dieser Metapher nach dem Modell eines in Brand gesetzten Gegenstandes, vorstellen.

\begin{tabular}{|l|c|l|}
\hline Deutsch & Serbisch & Äquivalenz \\
\hline das Herz entzünden & $/$ & Nulläquivalenz \\
\hline das Herz ist entflammt & $/$ & Nulläquivalenz \\
\hline$/$ & izgori kome srce za kim/čim & Nulläquivalenz \\
\hline
\end{tabular}

Phraseologismen das Herz entzünden (Liebe und Leidenschaft entfachen) und das Herz ist entflammt (er entbrennt in Liebe für jmdn) sind nur bei Röhrich

6 Die konzeptuelle Metapher LEBEN IST EIN GLÜCKSSPIEL laut Kövecses (2002: 72). 
(Röhrich1991-1992:704) bestätigt.

Der Phraseologismus izgori kome srce za kim/čim mit der Bedeutung große Sehnsucht nach jmdm haben ist nur im Serbischen belegt.

\subsection{LIEBE IST UNTEN}

Phraseologismen in dieser Gruppe stützen sich auf die Orientierungsmetapher LIEBE IST UNTEN. Zu den Orientierungsmetaphern zählen Lakoff und Johnson alle Metaphern, die abstrakte Dinge durch die Verwendung räumlicher Strukturierung beschreiben. Diese Metaphern basieren auf unserer physischen und kulturellen Erfahrung. Orientierungsmetaphern geben einem Konzept eine räumliche Beziehung, z.B. VERSTAND IST OBEN; GEFÜHL IST UNTEN (Lakoff/Johnson 2007:22-30).

In unserer Kultur hat der Mensch Tiere, Pflanzen und physische Umgebung unter seiner Kontrolle. DER MENSCH IST OBEN, also, VERSTAND IST OBEN, im Unterschied zum GEFÜHL IST UNTEN.

\begin{tabular}{|l|l|l|}
\hline Deutsch & Serbisch & Äquivalenz \\
\hline jmdm sein Herz zu Füßen legen & $/$ & Nulläquivalenz \\
\hline
\end{tabular}

\subsection{LIEBE IST PHYSISCHE NÄHE}

„Die Metaphorik der Liebe umfasst viele Bilder der Einheit und auch die verschiedenen Formen von Nähe, in denen die physikalische Qualität der Strecke für die Beziehung steht" (Schmitt 1995-1/1996:47-62). Menschen sehnen sich instinktiv nach der Nähe anderer Menschen, die sie lieben z.B.:

\begin{tabular}{|l|l|l|}
\hline Deutsch & Serbisch & Äquivalenz \\
\hline jmds Herz hängt an jmdm/etw & $/$ & Nulläquivalenz \\
\hline sein Herz an jmdn hängen & $/$ & Nulläquivalenz \\
\hline jmdm ans Herz gewachsen sein & prirasti k srcu/za srce nekome & Volläquivalenz \\
\hline \multicolumn{1}{|c|}{$/$} & sviti se oko srca nekome & Nulläquivalenz \\
\hline ein Herz und eine Seele sein & biti jedno telo i jedna dusa & Volläquivalenz \\
\hline
\end{tabular}

Nach Müller „ist das Herz als Sinnbild des Innersten des Menschen 
geeignet, Zuneigung und Begierde der Nähe und der Verbindung auszudrücken“ (Müller 2005:252). Der Phraseologismus ein Herz und eine Seele sein hat die Bedeutung unzertrennlich/ befreundet sein. Diese idiomatische Wendung betont „die Gesamtheit der Empfindungen und geistigen Kräfte“ (Duden 11 1998: 327) und beruht auf Apostelgeschichte 4,32. (Röhrich 1991-1992:707).

Der Somatismus Herz aus dem Herkunftsbereich ,steht für das Gefühl, die Seele, für die Meinung oder Gesinnung. Zusammengenommen soll damit eine Übereinstimmung in allen wesentlichen Bereichen ausgedrückt werden“ (Müller 2005:248).

\section{ZUSAMMENFASSUNG}

In dieser Arbeit wurde auf der Grundlage der kognitiven Metapherntheorie untersucht, nach welchen Konzepten deutsche und serbische somatische Phraseologismen im semantischen Feld LIEBE kategorisiert werden. Sie werden in ihrer Bildlichkeit verglichen. Der Untersuchungskorpus besteht aus deutschen und serbischen Somatismen, die aus entsprechenden ein- und zweischprachigen Wörterbüchern exzerpiert wurden. Es hat sich gezeigt, dass das Konzept der Liebe in beiden Sprachen ähnlich dargestellt wird. Den untersuchten Liebes-Somatismen liegen folgende konzeptuelle Metaphern zugrunde: LIEBE IST EIN OBJEKT (LIEBE IST EIN WERTVOLLES OBJEKT IN EINEM GEFÄSS, LIEBE IST ETWAS VERSTECKTES/EIN VERSTECKTES OBJEKT IN EINEM GEFÄSS, LIEBE IST EIN GESCHENK, LIEBE IST DAS STEHLEN EINES KOSTBAREN OBJEKTS, LIEBE IST EIN OBJEKT ZUM EROBERN, LIEBE IST DER FLUG EINES VOGELS, LIEBE IST EINE PFLANZE/BLUME, LIEBE IST EIN GLÜCKSSPIEL, LIEBE IST FEUER, LIEBE IST UNTEN und LIEBE IST PHYSISCHE NÄHE. Der hohe Grad an Übereinstimmungen mag wohl aus dem universellen Charakter der dargestellten Erscheinungen resultieren. Ein wichtiger Grund für die Gemeinsamkeiten beider Sprachen besteht darin, dass die Menschen den gleichen Natursetzen unterliegen. Viele konzeptuelle Metaphern sind nicht kulturspezischer, sondern übereinzelsprachlicher Art. Einige Metaphern können sogar als universell bezeichnet werden. Phraseologismen und ihre Bildwelt sind nicht so fremd, wenn sie konzeptuell untersucht werden. 


\section{LITERATUR}

Dobrovol'skij, D. (1997). Idiome im mentalen Lexikon. Trier: Wissenschaftlicher Verlag.

Duden 11 (1998). Redewendungen und sprichwörtliche Redensarten. Mannheim, Leipzig, Wien, Zürich: Dudenverlag.

Földes, C. (1985). Über die somatischen Phraseologismen der deutschen, russischen und ungarischen Sprache. Versuch einer konfrontantiven Analyse. In: Germanistisches Jahrbuch DDR-UVR/Lektorat für deutsche Sprache und Literatur beim Kultur- und Informationszentrum der DDR in Budapest. S.18-40.

Kövecses,Cz. (1999). Metaphor and Emotion. Language, Culture and Body in Human Feeling. Cambridge: Cambridge University Press.

Kövecses, Z. (2002). Metaphor: A Practical Introduction. Oxford and New York: Oxford University Press.

Lakoff, G. (1987). Women, fire and dangerous things: what categories reveal about the mind. Chicago: Chicago University Press.

Lakoff, G./Johnson, M. (2007). Leben in Metaphern. Konstruktion und Gebrauch von Sprachbildern.Übersetzung der deutschen Ausgabe von Astrid Hildebrand. Heidelberg, Fünfte Auflage.

Matešić, J. (1982). Frazeološki rječnik hrvatskoga ili srpskoga jezika. Zagreb: Školska knjiga.

Mrazović, P./ Primorac, R. (1991). Nemačko - srpskohrvatski frazeološki rečnik. Beograd: Naučna knjiga.

Müller, K. (2005). Lexikon der Redensarten, Herkunft und Bedeutung deutscher Redewendungen. Basserman Verlag.

Palm, Ch. (1997). Phraseologie. Eine Einführung. 2. Auflage. Tübingen: Narr.

Rečnik srpskog jezika. (2007). Novi Sad: Matica srpska.

Röhrich, L. (1991-1992). Das große Lexikon der sprichwörtlichen Redensarten. Band 1, 2, 3. Freiburg im Breisgau: Herder Verlag.

Sadikaj, S. (2010). Metaphorische Konzepte in somatischen Phraseologismen des Deutschen und Albanischen. Eine kontrastive Untersuchung anhand von Herz- und Hand-Somatismen. WespA. Würzburger elektronischen sprachwissenschaftlichen 
Arbeiten Nr. 9 (August 2010) http://www.spr.germanistik.uni-wuerzburg.de/wespa Schemann, H. (1991). Synonymwörterbuch der deutschen Redensarten. Stuttgart: Ernst Klett Verlag fur Wissen und Bildung, xxxvi, $428 \mathrm{~s}$.

Schmitt,R.(1/1995 -1/1996) Metaphernanalyse und die Repräsentation biographischer Konstrukte. In: Journal für Psychologie, Asanger-Verlag, Heidelberg, Doppelheft 1/1995 -1/1996, S. 47 - 62.

Schwarz-Friesel, M. (2007). Sprache und Emotion. Tübingen und Basel: A. Francke Verlag.

Gordana Ristić

\section{LOVE IN GERMAN AND SERBIAN IDIOMS}

\section{Summary}

The aim of the paper is to carry through a comparative study of somatic idioms in the semantic field of LOVE in German and Serbian language, on the basis of the cognitive and conceptual linguistic theory. The somatisms will be examined from the interlingual GermanSerbian aspect, with German being the source language, and Serbian the target language. The corpus has been excerpted from the relevant monolingual and bilingual idiom dictionaries.

Key words: idioms, emotions, love, contrastive and conceptual approach, conceptual metaphor.

Gordana Ristić

\section{LJUBAV U NEMAČKIM I SRPSKIM SOMATSKIM FRAZEOLOGIZMIMA}

\section{rezime}

Cilj ovog rada je kontrastivna analiza nemačkih i srpskih somatskih frazeologizama u semantičkom polju LJUBAV na osnovu kognitivne lingvističke teorije. Somatizmi su podvrgnuti interlingvalnoj kontrastivnoj analizi, pri čemu je polazni jezik nemački, a ciljni srpski. Korpus je ekscerpiran iz odgovarajućih frazeoloških rečnika oba jezika.

Ključne reči: frazeologizmi, emocije, ljubav, kontrastivna analiza, konceptualna metafora. 